\title{
Should peritoneal dialysis catheters be removed at the time of kidney transplantation?
}

\author{
Jeff Warren, MD; Emily Jones, ${ }^{*}$ Alp Sener, MD; ${ }^{*}$ Martin Drage, MD; ${ }^{*+}$ Ali Taqi, MD; ${ }^{*}$ Sian Griffin, MD; ${ }^{\dagger}$ Christo- \\ pher Watson, MD; ${ }^{\dagger}$ Patrick P. W. Luke, MD, FRCSC
}

*Multiorgan Transplant Program, London Health Sciences Centre, Department of Surgery, Schulich School of Medicine and Dentistry, Western University, London, ON; †University Department of Surgery, Addenbrooke's Hospital and the NIHR Cambridge Biomedical Research Centre, Cambridge, UK

See related article on page 379 .

Cite as: Can Urol Assoc J 2012;6(5):376-8. http://dx.doi.org/10.5489/cuaj.12112

\section{Abstract}

Background: Delayed graft function (DGF) following transplantation necessitates support in the form of hemodialyis (HD) or peritoneal dialysis (PD). However, post-transplant PD-related complication and failure rates are unknown.

Methods: We studies patients who were on PD at the time of kidney transplantation over a 4-year period at two separate institutions.

Results: Of the 137 PD patients, 19 had their catheters removed at the time of transplant. Of the remaining 118 patients, $89 \%$ had immediate graft function. PD-related complications in this group included peritonitis $(n=5)$, catheter-related infections $(n=2)$ and emergency laparotomy $(n=1)$. Of the 15 patients requiring posttransplant PD, 33\% developed peritonitis and 20\% had fluid-leaks necessitating HD. Overall, leaving a PD catheter in situ post- transplantation is associated with $7 \%$ rate of peritonitis versus $0 \%$ if removed $(p<0.05)$.

Conclusions: PD catheter removal should be considered at the time of renal transplantation, as postoperative PD-related failure/ complication rates are high.

\section{Introduction}

Prior to renal transplantation, many patients have been maintained on peritoneal dialysis (PD) as a form of renal replacement therapy. At the time of transplant, the European Best Practice Guidelines state that PD catheters not be removed in the event of delayed graft function (DGF) to facilitate continued renal support. ${ }^{1}$ However, apart from small case series in the pediatric transplant literature, the complication and failure rates related with postoperative PD are unknown. ${ }^{2-4}$

We hypothesized that use of PD catheters in transplant patients postoperatively leads to a high rate of failures due to lack of integrity of the peritoneum, as well as a high risk of infection. We review our experience with PD catheter management at two transplant centre to determine rates of infection and postoperative complications in kidney transplant recipients. This contemporary study aims to discover whether PD catheter removal at the time of surgery should be considered in patients undergoing renal transplantation.

\section{Methods}

The current study was approved by the ethics review boards of Western University and Addenbrooke's Hospital; it was conducted according to the Declaration of Helsinki protocols. Using a retrospective case-control assessment, PD failures and PD-related complications were evaluated from patient records.

During the 3-year period (2005-2008), 423 patients received kidney transplants at the study sites of the London Health Sciences Centre in London, Ontario, Canada and Addenbrooke's Hospital in Cambridge, UK. Of these patients, $137(32 \%)$ were treated with PD prior to renal transplantation. All patients not receiving PD prior to transplantation were excluded from the study. Of the 137 patients, $118 \mathrm{had}$ their PD catheters left in situ following renal transplantation; the remaining 19 patients had their catheters removed at the surgeon's discretion at the end of the renal transplant procedure.

Differences between groups were evaluated using the Chi-square test; $p<0.05$ was considered statistically significant.

\section{Results}

Of the 137 patients comprising the study group, 19 had their catheters removed at the time of transplant (Fig. 1). Of these 19 patients, 18 patients (95\%) had immediate graft function following transplantation and did not require dialysis at any point post-transplant. Only 1 patient (5\%) had DGF 
and required hemodialysis (HD) for 1 week postoperatively. Importantly, no complications arose as a result of HD access or therapy. None of these patients developed postoperative peritonitis, hernias, wound infections or any other complications related with concurrent catheter removal.

Of the 118 patients who had PD catheters left in situ post-transplant, 103 had immediate graft function (89\%). Despite not having dialysis performed, PD-related complications occurred in $6.8 \%(n=6)$ of transplants; 5 had peritonitis $(4.2 \%), 2(1.9 \%)$ had catheter exit-site infections and 1 $(1.0 \%)$ required an emergency laparotomy for "peritonitis" 2 months post-transplant (Fig. 1) Interestingly, laparotomy disclosed no evidence of intra-abdominal pathology, and a presumed diagnosis of "PD-related peritonitis" was made.

Following transplantation, $15(12.7 \%)$ patients with retained PD catheters required postoperative peritoneal dialysis for DGF. In this group, 5 patients (33.3\%) developed bacterial peritonitis and $3(20.0 \%)$ had dialysate-derived fluid-leaks from their incisions necessitating cessation of PD and initiation of HD (Fig. 1). In total, complications as a result of PD catheter use occurred in over $50 \%$ of patients; this number is significantly higher than in those cases in which PD catheter was removed or not used $(p<0.001)$.

In total, the rate of PD catheter-related complications was $12.7 \%$ when it was left in situ versus $0.0 \%$ when the catheter was removed at the time of surgery $(p=0.09)$. Accordingly, in the patients with immediate graft function, the PD catheter related complication rate was still $6.8 \%$ when the catheter was left in situ versus $0.0 \%$ when it was removed at the time of transplant ( $p=$ not significant). Therefore, even in patients

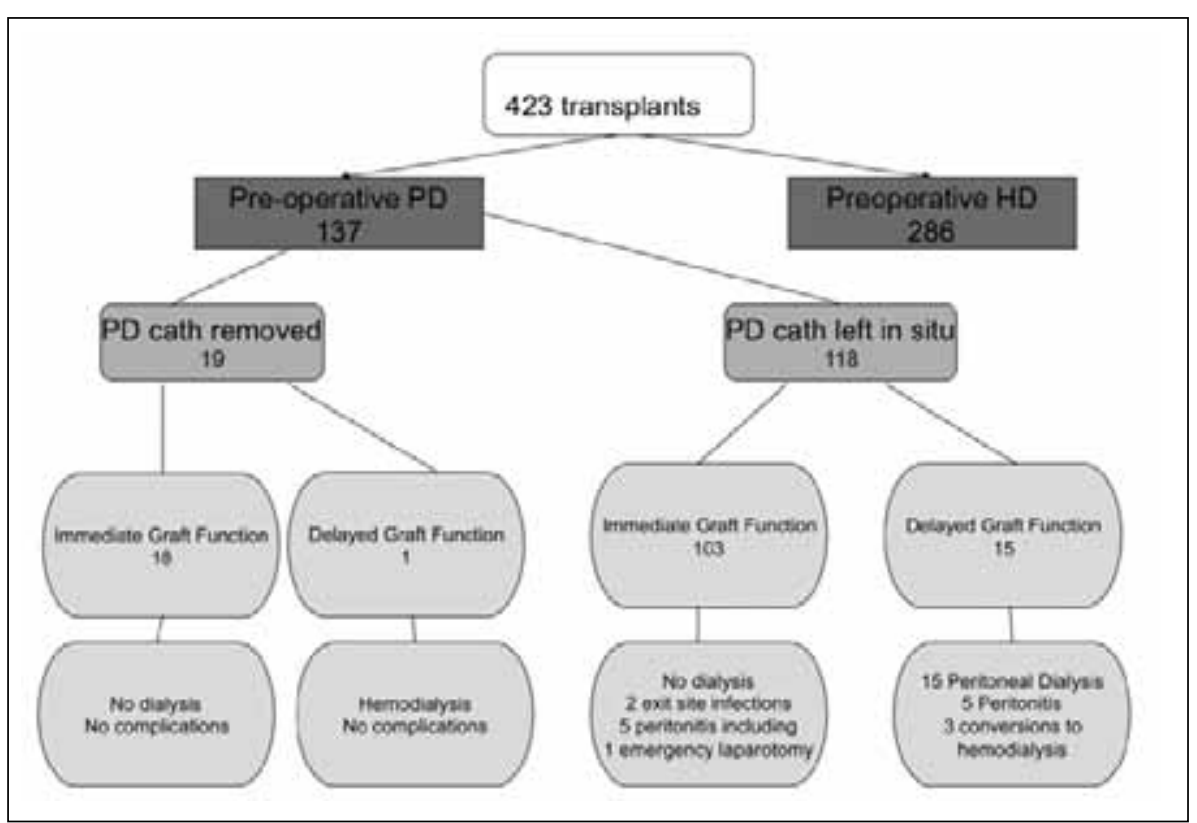

Fig. 1. Flow chart of peritoneal dialysis (PD) catheter-related outcomes from kidney transplant recipients treated with PD prior to transplantation; IGF: immediate graft function; DGF: delayed graft function; HD: hemodialysis. with a relatively low risk of postoperative DGF, there is a small, but finite, risk of catheter-related complications.

\section{Discussion}

Renal transplantation offers a significant lifestyle and mortality advantage over both hemodialysis and PD in patients with end-stage renal disease (ESRD). Although as many as $20 \%$ of ESRD patients are on PD at the time of transplantation, there has been no consensus with regards to the optimal timing of PD catheter removal. ${ }^{5}$ In the current study, we report that PD catheters left in situ following transplantation are associated with a higher risk of catheter-related complications compared with PD catheters removed at the time of renal transplantation, regardless of allograft function or need for postoperative PD.

The literature surrounding the timing of removal of PD catheters is sparse and mostly based on pediatric transplant studies. Arbeiter and colleagues demonstrated that of the 26 PD dependent patients, all of whom had their PD catheters left in situ following renal transplantation, 12 (46\%) required its use for DGF within the first month after the operation. ${ }^{2}$ Unfortunately, of these patients, $6(50 \%)$ had catheter-related peritonitis. All patients eventually required a second operation to have the PD catheter removed. ${ }^{2}$ Similarly, Palmer and colleagues clearly demonstrated that $43 \%$ of allograft recipients, who had PD catheters left in situ developed catheter-related infections, despite being treated with prophylactic antibiotics - all within the first 3 months post-transplant. Interestingly, the PD catheter was not used in any of these patients. ${ }^{4}$ Based on their findings, the authors have now mandated that PD catheters be removed prior to patients being discharged following transplantation, unfortunately necessitating a second anesthetic. Conversely, Andretta and colleagues showed that although PD catheters were left in place in all 80 graft recipients, there was only a $1 \%$ PD catheter-related infection in the first 3 months following the transplant; all patients subsequently underwent a second operation to have the catheters removed. ${ }^{3}$

These studies provide strong supporting evidence for our contemporary series in adult renal transplant recipients - leaving PD catheters in place following renal transplantation poses an appreciable risk of PD catheter-related complications, even if the catheter is not utilized. In fact, Gokald and Bakir's groups have separately reported that 
Warren et al.

although PD catheters may successfully be used in adults following renal transplantation, there is a high risk of emergent removal of the catheters due to non-resolving peritonitis or catheter-site infections even in patients who did not utilize PD access; removing the PD catheter at the time of the transplant may minimize these risks as well as mitigate the risks of a second anesthetic. ${ }^{6,7}$ Based on our findings, leaving a PD catheter in situ at the time of transplant has a $>50 \%$ chance of catheter-related complications if it is utilized and almost $7 \%$ if it was not. Additionally, PD was not technically feasible in $20 \%$ of patients due to leakage of dialysate from the wound, despite no appreciable violation of peritoneal cavity intraoperatively. This is compounded by the additional risks of a second anesthetic to remove the indwelling catheter at a later date. Based on these data, we have since modified our institutional policy. We now consider removing PD catheters at the time of renal transplantation with the caveat that, in the case of DGF, patients will require temporary hemodialysis (no line-related complications have been noted by our group thus far).

Our results are enlightening, but highlight the importance of further prospective studies that may delineate target populations of patients who would benefit from leaving PD catheters in situ, as well as defining the criteria by which the surgeon will remove the PD catheter (including risk stratification based upon donor status).

Although many centres leave PD catheters in situ at the time of renal transplantation, as recommended by the current guidelines for $\mathrm{PD}$ catheter management according to the European Best Practice Guidelines for PD, there is accumulating evidence that there is a high rate of PD catheterrelated complications when the catheters are left in situ.

\section{Conclusion}

The current study presents novel, contemporary results from the largest reported cohort of patients to suggest that PD catheter removal should be considered at the time of renal transplantation, as postoperative PD-related failure, infection and other complication rates are significant and can be associated with significant morbidity. Furthermore, performing the catheter removal at the time of the original surgery will circumvent the need for a second anesthetic in a patient who is recovering from a major operation.

Competing interests: None declared.

This paper has been peer-reviewed.

\section{References}

1. Dombros $N$, Dratwa $M$, Feriani $M$, et al. European best practice guidelines for peritoneal dialysis. 3 Peritoneal access. Nephrol Dial Transplant 2005;20:ix8-ix12.

2. Arbeiter K, Pichler A, Muerwald G, et al. Timing of peritoneal dialysis catheter removal after pediatric renal transplantation. Perit Dial Int 2001;21:467-70.

3. Andreetta B, Verrina E, Sorino P, et al. Complications linked to chronic peritoneal dialysis in children after kidney transplantation: experience of the Italian Registry of Pediatric Chronic Peritoneal Dialysis. Perit Dial Int 1996;16:S570-3.

4. Palmer JA, Kaiser BA, Polinsky MS, et al. Peritoneal dialysis catheter infections in children after renal transplantation: choosing the time of removal. Pediatr Nephrol 1994;8:715-8.

5. Chalem Y, Ryckelynck JP, Tuppin P, et al. Access to, and outcome of, renal transplantation according to treatment modality of end-stage renal disease in France. Kidney Int 2005;67:2448-53.

6. Gokal R, Kost S. Peritoneal dialysis immediately post transplantation. Adv Perit Dial 1999;15:112-5.

7. Bakir N, Surachno S, Sluiter WJ, et al. Peritonitis in peritoneal dialysis patients after renal transplantation. Nephrol Dial Transplant 1998;13:3178-83.

Correspondence: Dr. Patrick Luke, London Health Sciences Centre - University Campus, 339 Windermere Rd, London, 0N N6A 5A5; fax: 519-663-3858; patrick.luke@lhsc.on.ca 